Bundesgesundheitsbl 2018 61:1093-1102 https://doi.org/10.1007/s00103-018-2789-9 Online publiziert: 25 . Juli 2018

(c) Der/die Autor(en) 2018

CrossMark

Harald Dormann ${ }^{1} \cdot$ Renke Maas ${ }^{2} \cdot$ Christiane Eickhoff $^{3} \cdot$ Uta Müller $^{3}$. Martin Schulz ${ }^{3}$ Danny Brell ${ }^{4}$ Petra A. Thürmann ${ }^{5}$. MetropolMediplan 2016 . Modellregion Erfurt $\cdot$ PRIMA

'Zentrale Notaufnahme, Klinikum Fürth, Fürth, Deutschland

${ }^{2}$ Lehrstuhl für Klinische Pharmakologie und Klinische Toxikologie, Friedrich-Alexander-Universität Erlangen-Nürnberg, Erlangen, Deutschland

${ }^{3}$ Geschäftsbereich Arzneimittel, ABDA - Bundesvereinigung Deutscher Apothekerverbände e. V., Berlin, Deutschland

${ }^{4}$ Krankenhausapotheke, Helios Klinikum Erfurt, Erfurt, Deutschland

${ }^{5}$ Philipp Klee-Institut für Klinische Pharmakologie, Helios Universitätsklinikum Wuppertal, Lehrstuhl für Klinische Pharmakologie, Universität Witten/Herdecke, Wuppertal, Deutschland

\title{
Der bundeseinheitliche Medikationsplan in der Praxis
}

\section{Die Pilotprojekte MetropolMediplan 2016, Modellregion Erfurt und PRIMA}

nahme und der Grund der Einnahme der jeweiligen Medikation können ebenfalls auf dem BMP dokumentiert werden [3]. Durch den BMP ist ein multiprofessioneller und intersektoraler Austausch über die aktuelle Medikation eines Patienten möglich. Somit sind alle am Medikationsprozess Beteiligten über die aktuellen Arzneimittel idealerweise zum Zeitpunkt der Behandlung informiert. Dies kann zu einer Reduktion von Medikationsfehlern und zur Verbesserung der AMTS führen. Das 2015 verabschiedete E-HealthGesetz $\$ 31$ a und der Bundesmantelvertrag-Ärzte $\$ 29$ a regeln den Anspruch aller gesetzlich Krankenversicherten auf einen BMP, wenn diese gleichzeitig mindestens 3 verordnete Arzneimittel über mehr als 28 Tage einnehmen $[4,5]$.

In der aktuellen Versorgungsroutine wird der BMP vom Arzt auf Papier ausgedruckt und dem Patienten ausgehändigt. Zusätzlich zu den lesbaren Inhalten fasst ein 2-D-Barcode, der im rechten oberen Quadranten der Papierform automatisch mit aufgedruckt wird, alle Informationen elektronisch lesbar (Scanner) zusammen. Langfristig sollen die Inhalte des BMP auf der elektronischen Gesundheitskarte abgespeichert werden [4].

Im Rahmen der Aktionspläne wurden vom BMG drei Modellprojekte zur Er- probung eines Medikationsplans in der Praxis hinsichtlich Akzeptanz und Praktikabilität gefördert. Diese sind:

- MetropolMediplan 2016 in der Metropolregion Nürnberg/Erlangen/ Fürth (MMP16),

- Modellregion Erfurt (Erfurt),

- PRIMA (Primärsystem-Integration des Medikationsplans mit Akzeptanzuntersuchung) in der Modellregion Sachsen-Thüringen (PRIMA).

Dem vorangestellt wurde zunächst eine Lesbarkeits- und Verständlichkeitstestung des BMP mit Patientinnen und Patienten durchgeführt.

Lesbarkeits- und Verständlichkeitstestung des BMP

Diese Untersuchung erfolgte in strukturierten Interviews mit 40 multimorbiden Patientinnen und Patienten. Die Entwicklung des Interviewleitfadens basierte auf der Richtlinie der Europäischen Kommission zur Lesbarkeitstestung von Packungsbeilagen für die $\mathrm{Zu}$ lassung von Arzneimitteln [6]. Die Interviews beinhalteten zusätzlich eine praktische Übung zur Verständlichkeit, in der die Patientinnen und Patienten auf Basis eines Muster-BMP entsprechend der da- 
rin vorgegebenen Dosierungsvorschriften exemplarisch Dosetten mit Placebos befüllen sollten. Als objektives Bewertungsinstrument wurde das „Evaluation Tool to test the handling of the Medication Plan" (ET-MP) entwickelt [7].

Von den 40 befragten Patientinnen und Patienten fanden $26(65 \%)$ die Gestaltung des BMP übersichtlich. Die restlichen 14 Personen wünschten sich Vereinfachungen (Streichen von Spalten) oder Veränderungen in der Reihenfolge der Informationen. Fragen zur Orientierung auf dem BMP wurden von 38 Patientinnen und Patienten (96\%) richtig beantwortet. Jedoch zeigte die praktische Übung, dass bei 20 (50\%) der befragten Patientinnen und Patienten Unverständlichkeiten bezüglich der abgekürzten Tageszeitangaben der Spalte „Dosierung“ des BMP bestanden. Daraus resultierte die Anpassung dieser Beschriftung im BMP-Format dahin gehend, die Tageszeitangaben zukünftig auszuschreiben [8]. Weiterhin wurde die Empfehlung abgeleitet, komplexe Hinweise nicht auf die Spalten „Dosierung“ und „Hinweis“ aufzuteilen sowie Einnahmehinweise so konkret wie möglich $\mathrm{zu}$ formulieren, um die Verständlichkeit für Patientinnen und Patienten zu erhöhen.

In weiteren Untersuchungen mit insgesamt 140 Patientinnen und Patienten wurde die Verständlichkeit des gleichen Muster-BMP an je 50 Erkrankten mit der Primärdiagnose chronische Herzinsuffizienz (CHF), 50 Erkrankten mit der Primärdiagnose Diabetes mellitus Typ 2 (DM2) und 40 allgemein internistisch Behandelten untersucht $[9,10]$. Von den Befragten konnten $37 \%$ die gegebenen Informationen des Muster-BMP korrekt umsetzen. Gemessen wurde dies anhand eines „Evaluation Tool to test the handling of the Medication Plan" (ET-MP Score), der einen Cut-offvon $>90 \%$ (min. $0 \%$, max. $100 \%$ ) für das korrekte Umsetzen des Medikationsplans vorsieht.

\section{Die Modellprojekte in der Übersicht}

- Tab. 1 gibt eine zusammenfassende Übersicht der Fragestellungen, Metho- den, Patientenzahlen, technischen Umsetzungen und der Settings der jeweiligen Modellprojekte MMP16, Erfurt und PRIMA. Mehr Details der drei Projekte finden sich in den Kurz- und den ausführlichen Abschlussberichten unter: https:// www.bundesgesundheitsministerium. de/service/publikationen/gesundheit. html.

\section{MetropolMediplan 2016}

Am Projekt MetropolMediplan 2016 (MMP16) waren 12 Arztpraxen sowie 12 Apotheken aus Nürnberg und Fürth sowie das Klinikum Fürth, ein Klinikum der Schwerpunktversorgung, aktiv beteiligt. Es wurde die aus einem Vorprojekt (eMediPlan - sektorübergreifende Kommunikation von Medikationsdaten; Kapitel 07 03, Titel 686 60) bestehende und erprobte IT-Infrastruktur genutzt. Zusätzlich wurde ein 2-D-Barcodescanner zur Lesbarkeit der Papierversion des BMP implementiert [11]. In einem Vorprojekt wurden auf dem Markt erhältliche 2-D-Barcodescanner auf ihre Tauglichkeit getestet. Hierbei zeigte sich, dass nur ein Teil der als „HD-tauglich“ vermarkteten Scanner tatsächlich für das Einlesen des BMP geeignet war.

Das Projekt gliederte sich in zwei Stufen, von denen die zweite Stufe in drei Phasen unterteilt wurde. Erste Stufe: einfache Nutzung des BMP; Erstellung und Fortschreibung des BMP in der klinischen Versorgungsroutine. In der gesamten Laufzeit von zwei Jahren konnten 863 BMP durch Ärzte, Apotheker und das Klinikum ersterstellt werden (• Abb. 1) und 2070 BMP fortgeschrieben werden (• Abb. 2). Zweite Stufe: Hier wurde die Akzeptanz (1. Phase Einstellungsakzep$\operatorname{tanz}$, 2. Phase Handlungsakzeptanz und 3. Phase Nutzerakzeptanz) des BMP aus Sicht der Gesundheitsdienstleister (Ärztinnen und Ärzte, Apothekerinnen und Apotheker) sowie aus Sicht von ca. 110 Patientinnen und Patienten pro Studienphase evaluiert.

Es zeigte sich bei der Patientenbefragung über alle drei Interventionsphasen, dass ca. $20 \%$ der Patientinnen und Patienten ihre Medikation durch den Einsatz des BMP besser kannten und auch besser darüber informiert waren, wie sie ihre Medikation einnehmen sollten. Bezüglich der selbstberichteten Adhärenz (Einhalten der Therapieempfehlungen, Morisky-Score) fanden sich aber keine relevanten Änderungen über die Studienphasen. Gut $90 \%$ der befragten Patientinnen und Patienten fanden die Idee eines BMP gut. 95\% der Befragten stimmten dem Medikationsdatenaustausch unter den Gesundheitsdienstleistern zu. Dies resultierte nach Aussage der Patientinnen und Patienten aus einem Sicherheitsbedürfnis heraus, das durch einen aktuell gehaltenen BMP befriedigt wird (• Abb. 3). Bei der Befragung der Gesundheitsdienstleiter zeigte sich eine höhere Akzeptanz bei den beteiligten Apothekerinnen und Apothekern, insbesondere im Hinblick auf die Nutzenerwartung bezüglich der AMTS.

Die Mehrheit der befragten Apothekerinnen und Apotheker ( $80 \%$ ) erwartete, dass sich die Anzahl der Medikationsfehler reduzieren wird. Auf Seite der Ärztinnen und Ärzte rechneten jedoch nur 34\% mit einer Reduktion der Medikationsfehler. Das gleiche Bild zeigte sich bei der Beurteilung der Reduzierung unerwünschter Arzneimittelereignisse (UAE) durch die Nutzung des BMP. Hier schätzten $77 \%$ der Apothekerinnen und Apotheker und nur 29\% der Ärztinnen und Ärzte mit einer Reduktion von UAE durch den BMP. Auch bei der Einschätzung der Patientenzufriedenheit zeigte sich diese Diskrepanz der Berufsgruppen (• Abb. 4).

Bedingt durch das Studiendesign mit individueller Einwilligung der Patientinnen und Patienten, der Ärztinnen und Ärzte sowie der Apothekerinnen und Apotheker in die Projektteilnahme kann ein gewisses Bias hin zu einer positiven Grundeinstellung gegenüber dem BMP nicht ausgeschlossen werden. $\mathrm{Da}$ anzunehmen ist, dass jemand, der das Prinzip des BMP nicht mag, auch weniger wahrscheinlich an einer Studie zu dessen Erprobung teilnimmt.

Des Weiteren wurden die BMP hinsichtlich ihrer AMTS-Eignung untersucht. Bei 300 anonymisierten und zufällig ausgewählten BMP wurde eine Analyse auf Vollständigkeit (Wirkstoff, Handelsname, Stärke, Form, Dosierung, Einheit, Hinweise, Grund) und AMTS- 
Bundesgesundheitsbl 2018 61:1093-1102 https://doi.org/10.1007/s00103-018-2789-9

(c) Der/die Autor(en) 2018

\section{H. Dormann · R. Maas · C. Eickhoff · U. Müller · M. Schulz · D. Brell · P. A. Thürmann · MetropolMediplan 2016 · Modellregion Erfurt · PRIMA Der bundeseinheitliche Medikationsplan in der Praxis. Die Pilotprojekte MetropolMediplan 2016, Modellregion Erfurt und PRIMA}

\section{Zusammenfassung}

Seit dem 01.10.2016 haben gesetzlich Versicherte unter bestimmten Voraussetzungen einen Anspruch auf einen bundeseinheitlichen Medikationsplan (BMP, gemäß § 31a SGB V, E-Health Gesetz). Der Maßnahmenkatalog des 3. Aktionsplans 2013-2015 zur Verbesserung der Arzneimitteltherapiesicherheit (AMTS) sah die Erprobung des BMP in der Praxis hinsichtlich seiner Akzeptanz und Praktikabilität in drei Modellprojekten vor. Diese drei Projekte - MetropolMediplan 2016, Erfurt und PRIMA - werden vorgestellt und auf der Basis der gesammelten Erkenntnisse Empfehlungen abgeleitet. Der BMP wurde von den Teilnehmern in allen Projekten begrüßt und führte zu einem Anstieg der Patientenzufriedenheit und Verbesserung der
Medikationskompetenz. Sowohl die Ärzteals auch die Apothekerschaft bewerteten die interdisziplinäre und multiprofessionelle Zusammenarbeit über das Medium BMP sehr positiv. Negativ empfunden wurden der hohe zeitliche Aufwand zur Ersterstellung und Fortschreibung sowie die fehlende Telematikinfrastruktur zur Umsetzung einer elektronischen Version BMP. Ein Originaldatenabgleich der BMP-Daten mit den tatsächlich eingenommenen Medikamenten zeigte, dass lediglich bei $36 \%$ der Fälle der BMP und die tatsächlich eingenommenen Arzneistoffe übereinstimmten. Obwohl die Erstellung einer Papierversion des BMP nicht die Problematik der Aktualität und Vollständigkeit der Medikation zum
Behandlungszeitpunkt lösen konnte, wurde ein signifikanter Effekt auf die AMTS durch den BMP belegt. Neben Vorschlägen zur Verbesserung der Prozessintegration des BMP in die Versorgungsroutine der Ärzteund Apothekerschaft wird eine elektronisch sektorenübergreifende Verfügbarkeit des BMP gefordert. Der BMP sollte zukünftig ein zentrales und wichtiges Instrument zur Verbesserung der AMTS darstellen.

\section{Schlüsselwörter}

Arzneimitteltherapiesicherheit · Bundeseinheitlicher Medikationsplan · Pilotprojekte . Schnittstellen · Arzt-Apotheker-Kooperation

\section{Standardized national medication plan. The pilot projects MetropolMediplan 2016, model region Erfurt, and PRIMA}

\section{Abstract}

Since 1 October 2016, all legally insured persons are entitled to a nationwide medication plan (BMP) under certain conditions (according to § 31a SGB V, E-Health Law). The catalogue of measures of the 3rd Action Plan 2013-2015 for the improvement of drug therapy safety (AMTS) provided for the testing of a medication plan in practice, including its acceptance and practicability in three model projects. These three projects MetropolMediplan 2016, Erfurt, and PRIMA - are presented and recommendations are derived on the basis of the collected findings. Overall, the BMP was welcomed by the participating patients in all projects and led to an increase in satisfaction and an improvement in competence with regard to medication. Both doctors and pharmacists rated the interdisciplinary cooperation via the medium BMP very positively. The high effort and lack of technical infrastructure without electronic availability of the last current version of a BMP of the individual patient was perceived as negative. An original data comparison of the BMP data with the drugs actually taken in the MetropolMediplan 2016 project showed that only $36 \%$ of the patients were in agreement with the BMP and the drugs presently taken. The paper version of the BMP has therefore not yet been able to solve the problem of the timeliness and completeness of the medication. In addition to various proposals for the further development of BMP, all parties involved require the BMP to be available electronically across all sectors. The BMP should therefore be an important instrument for improving AMTS in the future.

\section{Keywords}

Medication safety - Medication plan

Pilot studies - Trans-sectoral care .

Physician/pharmacist cooperation
Tauglichkeit durchgeführt. Alle formalen Anforderungen erfüllten nur 19 (6,3\%) BMP. Als inhaltlich ausreichend vollständig für AMTS wurden 233 (77,7\%) BMP eingestuft. Ausreichend vollständig für AMTS heißt, dass beispielsweise der Wirkstoffname „Acetylsalicylsäure“, aber nicht der formal auch geforderte Markenname „Aspirin“, angegeben war. In 18 (6,0\%) AMTS-tauglichen BMP konnte mindestens ein hochwahrscheinlicher Medikationsfehler (Kontraindikationen laut Fachinformation) identifiziert werden.
Zum Verständnis, was vom BMP in Bezug auf die AMTS zu erwarten ist und was nicht, ist es wichtig zu berücksichtigen, dass der BMP primär mit dem Fokus einer adäquaten Information für Patientinnen und Patienten entwickelt wurde. Für die Gesundheitsdienstleister (Arztpraxis, Apotheke, Klinik) wäre ein digitales Kommunikationsmedium, ergänzt um weitere arztspezifische Informationen, essenziell, um das Potenzial für mehr AMTS ausschöpfen zu können und parallel zum BMP eine professionelle digitale Kommunikationskultur zu etablieren. Erste Erfahrungen wurden im Rahmen der Projekte eMediPlan und ARMIN (Arzneimittelinitiative SachsenThüringen) bereits gewonnen. Dennoch ist der BMP in der jetzigen Form bereits eine wichtige Basis für AMTS, da dieser trotz seiner Limitationen bereits durch die Gesundheitsdienstleister mitgenutzt wird.

Um den BMP und damit die Patientinnen und Patienten nicht mit Informationen $\mathrm{zu}$ überfrachten, sind einige für die objektive Beurteilung der AMTS durch die Gesundheitsdienstleister we- 
Tab. 1 Drei Modellprojekte zur Erprobung des bundeseinheitlichen Medikationsplans BMP. Vergleichende Übersicht über Methoden, Zielgrößen und Durchführung der geförderten Modellprojekte

MMP16 Erfurt

\section{Setting}

Teilnehmende Ge-

Ambulante und stationäre Versorgung

sundheitsdienst-

leister

Patientenzahlen

Einschluss

Patientenzahlen

Fortschreibung

Fragestellung 1

12 Arztpraxen, 12 Offizinapotheken Klinikum Fürth/Zentrale Notaufnahme

863

2070

Ambulante und stationäre Versorgung

12 Arztpraxen, 15 Apotheken, Helios

Klinikum Erfurt, $>10$ Stationen

161

130

Akzeptanz des BMP aus Sicht der Patientinnen und Patienten in der Versorgungsroutine

Fragestellung $2 \quad$ Akzeptanz des BMP aus Sicht der Gesundheitsdienstleister

Fragestellung 3

Auswertung der BMP hinsichtlich deren AMTS-Eignung

Fragestellung $4 \quad$ Vollständigkeit des BMP und Originaldatenabgleich

Methodik

Akzeptanz des BMP aus Sicht der Patientinnen und Patienten im Rahmen des Modellprojektes

Akzeptanz und Praktikabilität des BMP aus Sicht der Gesundheitsdienstleister ohne sektorenübergreifende IT-Infrastruktur

Auswertung der BMP hinsichtlich Anzahl der Medikationsprobleme

$-$

- Implementierung einer webbasier-
- In 3 Interventionsphasen Patientenbefragungen zur Akzeptanz

- In 3 Interventionsphasen Befragungen zur Akzeptanz für Gesundheitsdienstleister

- Bewertung der AMTS hinsichtlich der AMTS-Eignung in für die Versorgung typischen Situationen. Hierzu zählen:

- AMTS-Aspekte bei Ersterstellung und Fortschreibung (Änderung des BMP) und

- AMTS-relevante Situationen wie Präparateumstellungen

- Vergleich eines Medikationsdatenauszugs aus dem Praxisverwaltungssystem (PVS) mit dem aktuellen BMP. Vergleich des BMP mit dem Ergebnis der Brown-BagBefragung von Patientinnen und Patienten. Vollständigkeit und AMTS-Tauglichkeit der BMP auf Basis einer Zufallsstichprobe von 300 anonymisierten BMP
PRIMA

Ambulante Versorgung

12 Arztpraxen, 12 Offizinapotheken, die jeweils in Arzt-Apotheker-Teams zusammenarbeiten

196

196 ten Software für die teilnehmende Ärzte-/Apothekerschaft und das Krankenhaus

- Abstimmung der Prozesse und eines Berechtigungskonzeptes für den BMP

- Rekrutierung von Patientinnen und Patienten in der Klinik und in Hausarztpraxen

- Befragung der Patientinnen und Patienten nach 6 und nach 12 Monaten mittels standardisierter Fragebögen, MARS-D und SIMS-D

- Befragung der Gesundheitsdienstleister nach 6 Monaten (Krankenhauspersonal) bzw. 12 Monaten (Hausärzte- und Apothekerschaft)

- Analyse der Medikationspläne im Hinblick auf Medikationswechsel und arzneimittelbezogene Probleme
Umsetzung des BMP in die Versorgungsrealität von Ärztinnen und Ärzten, Apothekerinnen und Apothekern sowie Patientinnen und Patienten mit Untersuchung von Machbarkeit und Praktikabilität

Durchführung einer Akzeptanzbefragung bei Patientinnen und Patienten sowie bei der Ärzte- und Apothekerschaft

$-$

Rekrutierung von mind. 8 Arzt-Apotheker-Teams, die jeweils ca. 10 Patienten rekrutierten

Untersuchung von Machbarkeit und Praktikabilität der BMP-Erstellung und-Aktualisierung:

- Entwicklung und Erprobung der technischen Umsetzung und der Prozesse für die Erstellung der BMP in der Primärsoftware der Heilberufler sowie der Austausch der BMP über den Medikationsplanserver in einem mehrstufigen Prozess mit jeweiligen Feedbackzyklen:

- Phase 0: technische Testung (Austausch elektronischer BMP über den Server zwischen Primärsoftwareanbietern)

- Phase 1: Testfall (erstmalige Testung durch Ärzte- und Apothekerschaft)

- Phase 2: Testung der Software und Prozesse unter Alltagsbedingungen (je 2 Patientinnen und Patienten pro Arzt-Apotheker-Team)

- Phase 3: Routinebetrieb (je ca. 10 Patientinnen und Patienten pro Arzt-Apotheker-Team)

- Bewertung der Prozesse im Medikationsmanagement, der Arzt-Apotheker-Zusammenarbeit und des Patientennutzens durch die am Projekt teilnehmenden Ärzte und Apotheker in einem Workshop

- Durchführung von mind. 10 semistrukturierten Face-to-Face-Patienteninterviews

Schriftliche Akzeptanzbefragungen bei Ärztinnen und Ärzten, Apothekerinnen und Apothekern sowie Patientinnen und Patienten 


\begin{tabular}{|c|c|c|c|}
\hline & MMP16 & Erfurt & PRIMA \\
\hline $\begin{array}{l}\text { Technische } \\
\text { Umsetzung }\end{array}$ & $\begin{array}{l}\text { - In } 12 \text { Arztpraxen, } 12 \text { Apotheken } \\
\text { sowie im Klinikum Fürth wurde die } \\
\text { Anwendersoftware „eMediPlan“ in- } \\
\text { stalliert und entsprechende bereits } \\
\text { im Vorprojekt getestete Scannerty- } \\
\text { pen wurden etabliert } \\
\text { - Zentrale serverbasierte Datenspei- } \\
\text { cherung im Klinikum Fürth } \\
\text { - Implementierung eines serverba- } \\
\text { sierten BMP zum sektorübergrei- } \\
\text { fenden Austausch von BMP auf der } \\
\text { Basis von eMediPlan }\end{array}$ & $\begin{array}{l}\text { - Verfügbarkeit der Software und } \\
\text { Scanner in } 12 \text { Arztpraxen, } 15 \text { Apo- } \\
\text { theken, im Helios Klinikum Erfurt } \\
\text { - Zentrale serverbasierte Datenspei- } \\
\text { cherung in den Helios-Klinken }\end{array}$ & $\begin{array}{l}\text { - Elektronischer Datenaustausch zwischen } \\
12 \text { Arztpraxen und } 12 \text { Apotheken } \\
\text { - Integration eines neuen Softwaremoduls } \\
\text { in die Primärsysteme von Ärzten und } \\
\text { Apothekern } \\
\text { - Datenaustausch im KV-SafeNet via Medi- } \\
\text { kationsplanserver } \\
\text { - Dezentrale Datenspeicherung in Arztpra- } \\
\text { xen und Apotheken }\end{array}$ \\
\hline
\end{tabular}

sentliche Informationen auf dem BMP nicht oder nicht in AMTS-tauglicher Weise hinterlegt. Hierzu gehören therapie- und sicherheitsrelevante Diagnosen, die z.B. Kontraindikationen zu einer neuen Medikation bedingen, oder auch die Historie von unerwünschten Arzneimittelwirkungen. Der „Einnahmegrund“ kann hier zwar wichtige Hinweise geben, ist aber oft wegen seines Patientenfokus aus AMTS-Sicht zu ungenau formuliert - z.B. „Herz“ statt „Herzinsuffizienz NYHA III“. Therapierelevante Diagnosen, die z. B. eine exakte Dosisreduktion bedingen, würden nicht auf dem BMP erscheinen.

Weiterhin zeigte sich, dass die kollektive, sektorenübergreifende Pflege von BMP durch Ärzteschaft und Apothekerschaft, also die gemeinsame und lückenlose Fortschreibung der BMP, die formale Vollständigkeit und AMTSTauglichkeit der BMP bezogen auf die Einzelpräparate verbesserte. Dadurch erhöhte sich auch die Informationsqualität für Patientinnen und Patienten. Ein Originaldatenabgleich, der in den Apotheken mithilfe des „Brown-BagVerfahrens" (Methode zur strukturierten Erfassung der gesamten Medikation; die Patientinnen und Patienten bringen ihre gesamte Medikation in einer Tüte mit in die Apotheke) erhoben wurde, zeigte, dass nur bei $36 \%$ der befragten Patientinnen und Patienten die im BMP hinterlegten Arzneimittel vollständig mit denen laut Brown-Bag tatsächlich eingenommenen übereinstimmten. Bei $32 \%$ fanden sich im BMP mehr Medikamente als in der Brown-Bag, bei weiteren $32 \%$ zeigte die Brown-Bag weniger Arz- neimittel als auf dem BMP aufgelistet waren.

\section{Modellregion Erfurt}

Im Projekt „Erfurt“ wurden die Anwendung und der Austausch des BMP zwischen Krankenhaus (Helios Klinikum Erfurt), 12 niedergelassenen Hausarztpraxen und 15 Apotheken erprobt. Für das Projekt konnten die BMP mittels 2-DBarcodescanner eingelesen und über eine webbasierte Software modifiziert werden. Eine elektronisch gespeicherte Version stand den Teilnehmern nicht zur Verfügung, sondern konnte nur zu Auswertungszwecken verwendet werden. Somit spiegelt sich die Versorgungsrealität wider, mit allen Fallstricken des Vergessens und handschriftlicher Ergänzungen. Die insgesamt 161 eingeschlossenen Patientinnen und Patienten wurden über ein Jahr lang betreut. Relevante Zielgrößen waren Patientenzufriedenheit, selbstberichtete Adhärenz sowie Akzeptanz und Zufriedenheit seitens der Gesundheitsdienstleister.

Eingeschlossen wurden 161 Patientinnen und Patienten (59\% Frauen) mit einem mittleren Lebensalter von $69 \pm 9$ Jahren. Im Median befanden sich zu Beginn 8 verschiedene Wirkstoffe auf dem ersten Medikationsplan, nach 6 Monaten kam es zu einem Anstieg auf 10 Wirkstoffe $(p<0,001)$. Analysiert man die Veränderungen der Medikation auf Wirkstoffebene, so kam es durchschnittlich zu drei Veränderungen pro Patient in 12 Monaten.

Knapp $92 \%$ der Patientinnen und Patienten hielten den BMP nach einem Jahr für eine gute Idee, sie fanden ihn ver- ständlich und übersichtlich. Nahezu $85 \%$ fühlten sich nach einem Jahr Anwendung sicherer im Umgang mit ihren Arzneimitteln und der Großteil (81 \%) der Patientinnen und Patienten wünschten sich am Ende des Projektes eine gemeinsame Pflege ihres BMP durch Ärzte- und Apothekerschaft. Die Zufriedenheit mit der Information über ihre Medikation zeigte bei den Wissensfragen eine signifikante Zunahme. Die selbstberichtete Adhärenz war schon zu Beginn des Projektes sehr hoch und konnte nicht gesteigert werden. Hausärztinnen und Hausärzte, Apothekerinnen und Apotheker sowie Klinikärztinnen und Klinikärzte waren dem BMP gegenüber ebenfalls positiv eingestellt und sahen den Nutzen für Patientinnen und Patienten. Die meisten empfanden den BMP als verständlich und übersichtlich. Es wurde jedoch als problematisch beurteilt, dass Patientinnen und Patienten oftmals ihre Pläne vergaßen und die BMP nicht zentral verfügbar waren. Auch beim Zeitfaktor waren die Gesundheitsdienstleister kritisch. Nur ein Drittel der Hausärztinnen und Hausärzte fand den Zeitaufwand für die Erstellung akzeptabel, ebenso empfand ein Drittel der Hausärztinnen und Hausärzte und $22 \%$ der Apothekerschaft die erforderliche Zeit für die Fortschreibung im Praxis- und Apothekenalltag als akzeptabel.

Die hohe Anzahl der dokumentierten Medikamente und die erheblichen Veränderungen innerhalb eines Jahres erklären, warum die Patientinnen und Patienten wiederum durch die vielen neu auszustellenden BMP teilweise etwas überfordert waren. 


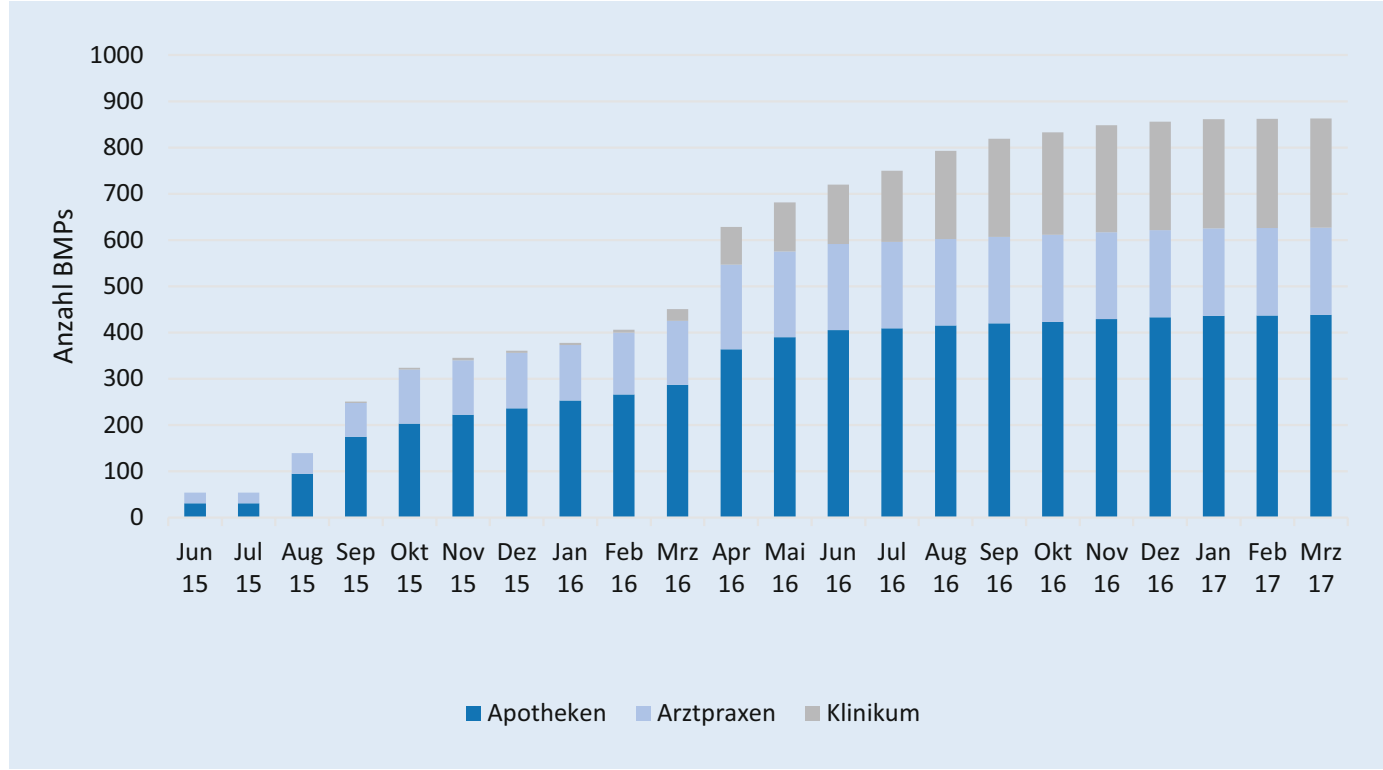

Abb. 14 Entwicklung der Gesamtzahl von BMP-Ersterstellungen im Verlauf der Studie MetropolMediplan $2016(N=863)$, Stand: 31.03.2017

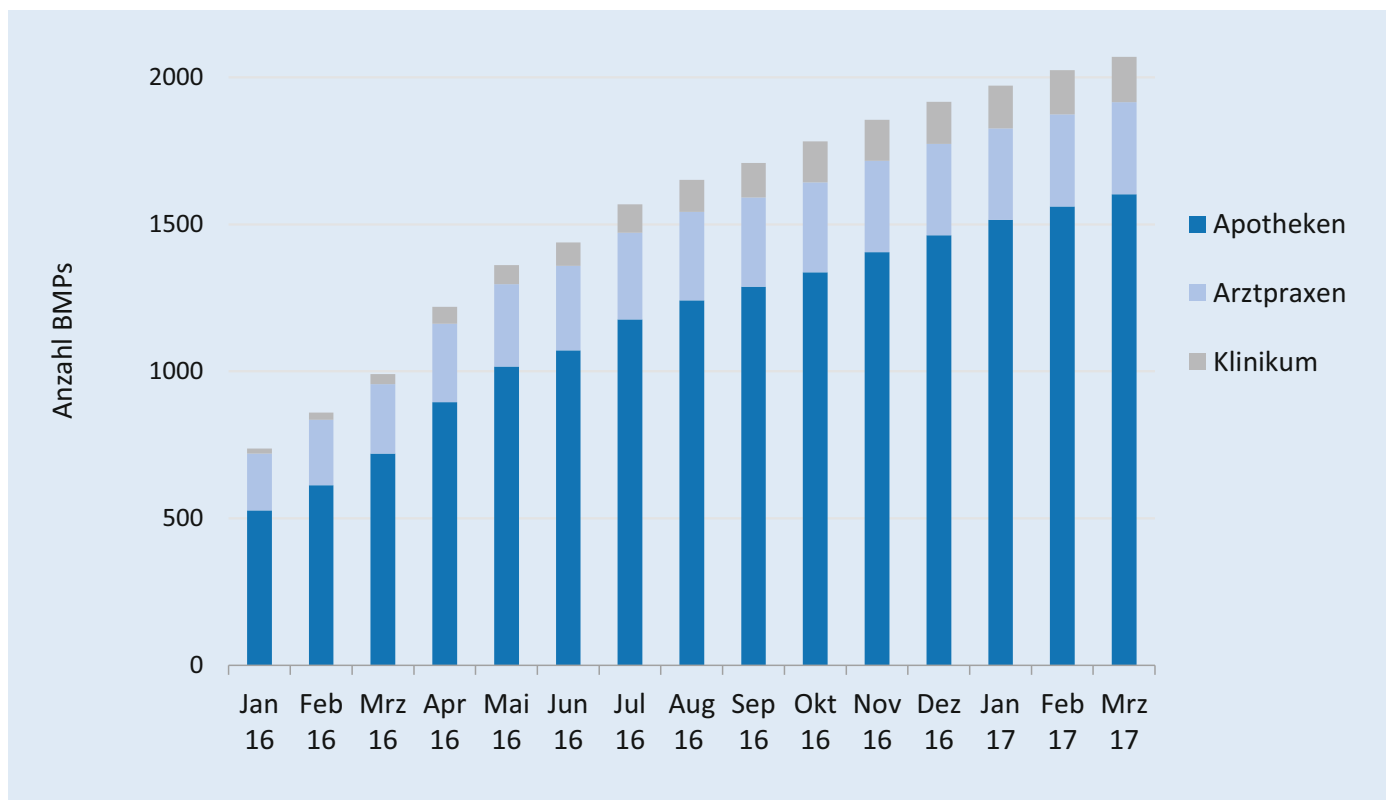

Abb. $2<$ Entwicklung der Gesamtzahl von BMPFortschreibungen im gesamten Verlauf der Studie MetropolMediplan $2016(N=2070)$, Stand: 31.03.2017

\section{PRIMA}

Ziel des Modellprojekts PRIMA war die Untersuchung der Praktikabilität und Akzeptanz eines gemeinsam von Ärztinnen und Ärzten sowie Apothekerinnen und Apothekern erstellten und gepflegten, elektronischen Medikationsplans im BMP-Format. Dieses Projekt wurde in den Bundesländern Sachsen und Thüringen durchgeführt.

Projektpartner waren die ABDA (Bundesvereinigung Deutscher Apothekerverbände e.V.), die Kassenärztliche Bundesvereinigung, die Landesapothe- kerverbände sowie kassenärztlichen Vereinigungen in Sachsen und Thüringen, die Freie Universität Berlin und die AOK PLUS.

Die Hauptuntersuchung beinhaltete die Implementierung des BMP durch Integration in das jeweilige Primärsoftwaresystem von Arztpraxen und Apotheken. Ziel war es, die Erstellung bzw. Aktualisierung von BMP und deren digitalen Austausch in den Versorgungsalltag zu integrieren und die Akzeptanz seitens der Ärztinnen und Ärzte, Apothekerinnen und Apotheker sowie Patientinnen und Patienten zu untersuchen.
Die Hauptuntersuchung wurde unter Beteiligung von vier Apothekenverwaltungssystemen sowie einem Praxisverwaltungssystem durchgeführt. PRIMA griff dabei auf die bestehende IT-Infrastruktur sowie festgelegte Zuständigkeiten der Ärztinnen und Ärzte und Apothekerinnen und Apotheker im Medikationsmanagement aus ARMIN (Arzneimittelinitiative SachsenThüringen, www.arzeimittelinitiative. de) zurück, die im Rahmen von PRIMA erstmals in der Praxis erprobt wurden. Diese Ergebnisse sind im Folgenden dargestellt: 


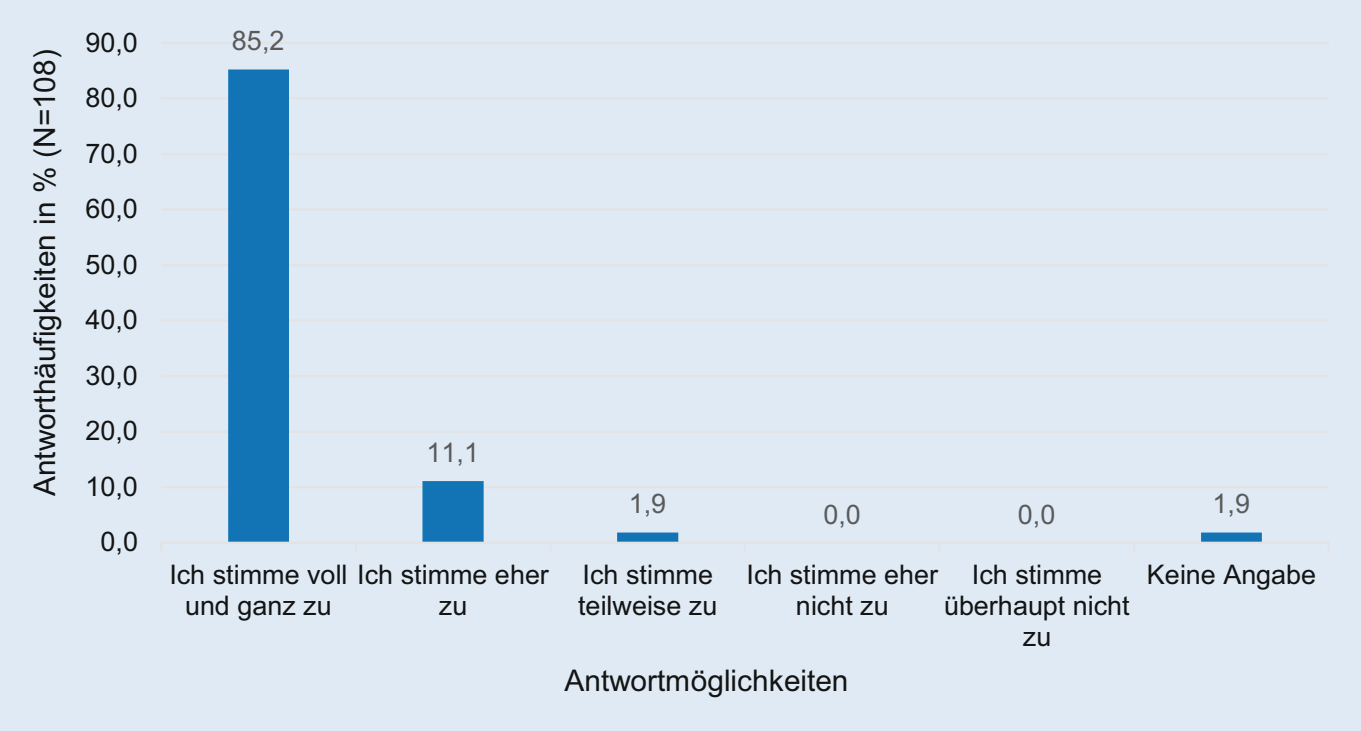

Abb. $3<$ Auswertung zur Frage: „Ich findees gut, dass meine Ärzte und Apotheker zu meiner Sicherheit meine Medikationsdaten kennen und diese regelmäßig pflegen, fortschreiben und untereinander Informationen zu meiner Medikation austauschen", im Rahmen der Studie MetropolMediplan 2016

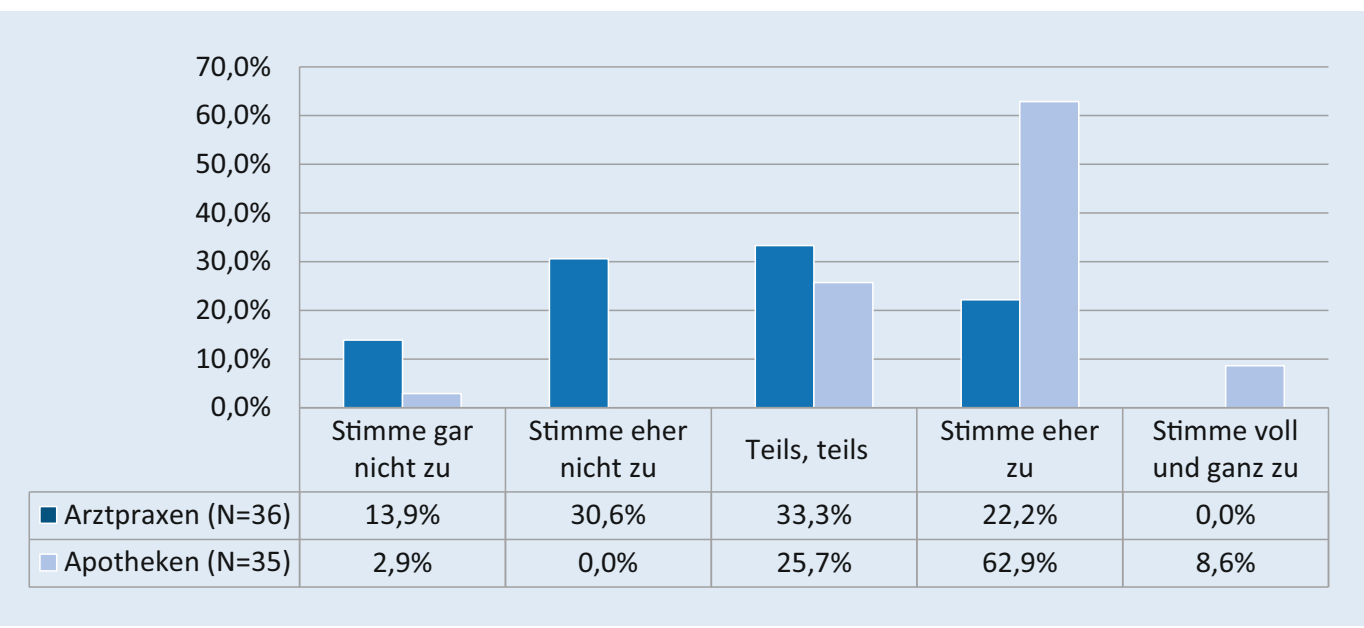

Abb. $4<$ Erwartete Verbesserung der Patientenzufriedenheit aus der Perspektive der Ärzte und Apotheker (Frage: „Aus meiner Erfahrung schätze ich, dass sich durch die Nutzung des BMP die Patientenzufriedenheit verbessert."). Studie: MetropolMediplan 2016

Über die Gesamtprojektlaufzeit von PRIMA stellte sich ein kontinuierliches Monitoring- und Supportkonzept zur Optimierung der komplexen technischen Strukturen als dringend erforderlich heraus. 11 Arzt-Apotheker-Teams durchliefen erfolgreich alle Phasen der praktischen Umsetzung und schlossen bis zum Ende des Förderzeitraums 196 Patientinnen und Patienten ein. Zunächst wurden die Richtigkeit und Vollständigkeit des Datenaustauschs hergestellt und getestet. Im Anschluss wurde die Machbarkeit der Erstellung und Pflege patientenindividueller BMP nachgewiesen. Die Benutzerfreundlichkeit der Softwareoberflächen und -funktionen wurde verbessert. Die definierten inhaltlichen Zuständigkeiten von Ärztinnen und Ärzten sowie Apothekerinnen und Apo- thekern im Medikationsmanagement trugen entscheidend zur Praktikabilität bei. In einem vierstündigen Workshop bewerteten 35 Teilnehmerinnen und Teilnehmer aus jeweils 10 Arztpraxen und Apotheken unterschiedliche Aspekte des Projektes. Anschließend wurden die Ergebnisse zusammengefasst und in strukturierten, moderierten Diskussionen bewertet. Über $80 \%$ bewerteten den Informationsaustausch während der Zusammenarbeit positiv, wobei den softwareunterstützten Kommentarfeldern zum BMP große Bedeutung für die fachlich-inhaltliche Kommunikation in der Routinebetreuung beigemessen wurde. Die Workshopteilnehmerinnen und -teilnehmer sahen sowohl einen persönlichen Nutzen im fachlichen Austausch untereinander als auch potenziell einen klinischen, humanistischen und ökonomischen Nutzen für Patientinnen und Patienten.

An der schriftlichen Akzeptanzbefragung nahmen 10 Ärztinnen und Ärzte, 8 Apothekerinnen und Apotheker und 103 Patientinnen und Patienten teil. Die Relevanz des BMP für die AMTS wurde von den Gesundheitsdienstleistern als Hauptgrund für eine Teilnahme am Projekt genannt. Etwa $75 \%$ der befragten Patientinnen und Patienten nahmen regelmäßig zwischen 5 bis 10 Arzneimittel ein und fast $20 \%$ wendeten regelmäßig 11 oder mehr Arzneimittel an. $70 \%$ nahmen zum Zeitpunkt der Befragung bereits $\geq 3$ Monate an PRIMA teil. Bei einer Projektteilnahme von über 3 Monaten hatten bereits ca. $60 \%$ der Patientinnen und Patienten mindestens einen neuen 
BMP erhalten. Etwa 2/3 der Patientinnen und Patienten gaben an, dass sich ihr Wissen, sowohl was die Dosierung als auch den Einnahmegrund der angewendeten Arzneimittel angeht, verbessert hätte. Knapp 70 \% fühlten sich durch die Betreuung sicherer im Umgang mit ihren Arzneimitteln. Für die meisten Patientinnen und Patienten ( $84 \%$ ) bestand jedoch der größte persönliche Nutzen im besseren Informationsaustausch der Gesundheitsdienstleister über die Medikation. Nur etwa $13 \%$ der Befragten fanden, dass der BMP im Hinblick auf die Wissensaspekte und die eigene Sicherheit im Umgang mit den Arzneimitteln einen besonderen Beitrag leisten würde; vielmehr stand der Betreuungsprozess im Vordergrund. Der häufigste Verwendungszweck des BMP war bei $70 \%$ der Patientinnen und Patienten die Mitnahme beim Besuch der Fachärztin oder des Facharztes. Etwa die Hälfte (51\%) verwendete den BMP regelmäßig bei der Arzneimittelanwendung, während $26 \%$ angaben, ihn hin und wieder als Erinnerung hinzuziehen.

\section{Abgeleitete Empfehlungen aus den drei Modellprojekten}

- Prozesse, Zuständigkeiten und Verantwortlichkeiten von Ärzte- und Apothekerschaft bzgl. der Ersterstellung und Fortschreibung des BMP müssen klar geregelt werden - Verfahrensanweisungen sind zu konkretisieren.

- Der BMP muss in die Primärsoftware (Apothekenverwaltungssystem, Krankhausinformationssystem und Praxisverwaltungssystem) integriert sein.

- Für den BMP müssen einheitliche Qualitätsstandards und -anforderungen, v. a. an die Software, festgelegt werden.

- Eine verpflichtende Beteiligung der Apotheken sowohl bei der Ersterstellung als auch bei Fortschreibung des BMP ist notwendig.

- Eine Papierversion des BMP für die Patientinnen und Patienten wird weiterhin gewünscht.

- Eine Integration des BMP in eine elektronische Patientenakte als vollintegraler Bestandteil mit entsprechender Zugriffsverwaltung ist zur lückenlosen Dokumentation essenziell. Parallelitäten und dadurch verursachte Differenzen von Medikationsinformationen zwischen einer elektronischen Gesundheitskarte, $\mathrm{Pa}$ tientenakte und dem Notfalldatensatz müssen vermieden werden.

- In zukünftigen elektronischen BMP sollen auch Therapie- und AMTS-relevante Diagnosen sowie die Historie unerwünschter Arzneimittelwirkungen hinterlegbar sein.

- Die Kliniken müssen lückenlos in den Medikationsprozess integriert werden. Idealerweise erhält jeder Patient mit einer Krankenhauseinweisung einen aktuellen BMP oder eine Aktualisierung des bestehenden BMP durch den Einweiser.

- Eine hohe Qualität des BMP/eMP (elektronischer Medikationsplan) ist nur durch eine zeitlich aufwendige und intellektuelle Auseinandersetzung mit der Medikation eines Patienten bei angemessener Aufwandsentschädigung realisierbar.

- Der Betreuungsprozess ist entsprechend den individuellen Anforderungen der Patientinnen und Patienten zu etablieren.

- Der Nutzen des BMP, der zwischen Ärztinnen und Ärzten sowie Apothekerinnen und Apothekern auf Basis einer Medikationsanalyse (z. B. Brown-Bag-Verfahren) abgestimmt wird, und v. a. der Nutzen dieses Prozesses müssen der Zielgruppe besser bekanntgemacht und vermittelt werden.

- Der Patient darf nicht Bittsteller eines BMP sein, wie im $₫ 31$ a SGB V, E-Health Gesetz vorgesehen, sondern der BMP sollte jedem Patienten, der Anspruch darauf hat, aktiv durch den Arzt oder Apotheker angeboten werden.

\section{Vorschläge zur Weiter- entwicklung des BMP}

Die Ergebnisse der 3 Modellprojekte wurden im Mai 2017 im Rahmen eines Workshops dem BMG, der Koordinierungsgruppe AMTS und den Vertragspartnern nach $\$ 31$ a Absatz 4 SGB V vorgestellt. Ziel war eine Diskussion zur Weiterentwicklung des BMP, wie der Aktionsplan 2016-2019 zur Verbesserung der AMTS in Deutschland dies in Maßnahme 22 vorsieht [12].

Nachfolgend werden, basierend auf den Erfahrungen und Ergebnissen der Modellprojekte, 5 zwischen den Projektbeteiligten abgestimmte Vorschläge zur Weiterentwicklung des BMP dargestellt.

Vorschlag 1: Implementierung einer codierten Wirkstoffverordnung

Aktuelle Situation. Als Standardcodierung der Arzneimittelangaben werden im BMP aktuell die Pharmazentralnummern (PZN) der Fertigarzneimittel verwendet (daraus werden weiterführende Angaben, wie z.B. Wirkstoff und Stärke, aus den jeweiligen Arzneimitteldatenbanken hergeleitet); abweichende/andere Angaben können ausschließlich als Freitexte hinterlegt werden und benötigen dadurch entsprechend viel Speicherplatz im Barcode.

Ziel. Erstellung und kontinuierliche Weiterpflege eines Standarddatensatzes zur PZN-unabhängigen codierten Beschreibung von Wirkstoffverordnungen, der über alle Arzneimitteldatenbanken hinweg verfügbar ist; entsprechende Berücksichtigung im Rahmen einer künftigen BMP-Fortschreibung.

\section{Vorschlag 2: Definition von Standardprozessen und Zuständigkeiten im Rahmen der Ersterstellung und kontinuierlichen Pflege von BMP bzw. eMP}

Aktuelle Situation. Der Schwerpunkt der getesteten BMP-Version (V 2.3) liegt ebenso wie bei der seit 01.10.2017 gültigen Version (V 2.4) in der Beschreibung der Datenfelder des BMP sowie ihrer korrekten Darstellung im Papierausdruck (Layout). Prozesse, Abläufe und Zuständigkeiten der unterschiedlichen Akteure werden im Rahmen der technischen Beschreibungen zwar skizziert, stellenweise wurde ihre umfassende und eindeutige Beschreibung jedoch 
zurückgestellt oder als unverbindliche Empfehlungen formuliert. Auch die entsprechenden Dokumentationen der Gesellschaft für Telematikanwendungen der Gesundheitskarte mbH (Gematik) fokussieren im Wesentlichen technische (und weniger inhaltliche) Prozesse und Zuständigkeiten. Da die Modellprojekte inzwischen erforderliche Prozesse und Erfordernisse aufgezeigt haben, sollten diese Erkenntnisse bei zukünftigen Implementierungen berücksichtigt werden.

Obwohl die Modellprojekte Prozesse zur Ersterstellung und Fortschreibung des BMP erfolgreich implementieren konnten, müssten noch einige praktische Aspekte zwischen den Akteuren (Ärztin/Arzt, Apothekerin/Apotheker, Patientin/Patient) klarer definiert werden. Beispiele sind:

- Wer darf bzw. muss den Medikationsplan wann und wie ändern? Wann soll beispielsweise ein neues Medikament eingetragen werden - sobald es die Hausärztin/der Hausarzt verordnet oder erst wenn die Patientin/der Patient das Rezept (Tage später) in der Apotheke auch tatsächlich einlöst? Wer trägt die Medikation ein, welche die Patientin/der Patient ohne ärztliche Verordnung in der Apotheke erwirbt?

- Wie sieht es bei der Ersterstellung des Medikationsplans mit Angaben der Patientin/des Patienten zur Medikation durch Fachkolleginnen/-kollegen aus? Tragen die Hausärztin/der Hausarzt diese direkt ein (oder ggf. nach welcher Form von Überprüfung)?

- Wie intensiv muss/kann eine fortschreibende Ärztin oder ein fortschreibender Arzt, die/der einen Wirkstoff ergänzt, prüfen, ob der Medikationsplan bezüglich der anderen Medikamente vollständig und aktuell ist? Hier klaffen die in der klinischen Versorgung praktizierte Verordnungsroutine und die notwendige analytische Sorgfalt oft auseinander, was es sinnvoll macht, auch haftungsrechtliche Verantwortlichkeiten $\mathrm{zu}$ klären.

- Entstehen Gesundheitsschäden durch fehlerhafte Einträge, mangelnde Vollständigkeit oder ignorierte Medikationsfehler? Der Apothekerin/dem
Apotheker fällt beispielsweise anhand neuer Informationen ein Medikationsfehler in der Medikation der Hausärztin/des Hausarztes auf. Wie und ggf. wie schnell sind Hausärztin/ Hausarzt und Patientin/Patient zu informieren, was ist aktuell bezüglich der Medikation zu tun, wenn der Hausarzt nicht erreichbar ist? Wer haftet, wenn der Fehler nicht zügig behoben wird?

Zusammengefasst werden Transparenzregeln benötigt, die den Verkehr des BMP zwischen den Akteuren regeln.

Ziel. Die Abstimmung der Prozesse und Zuständigkeiten in der Erstellung und Fortschreibung der BMP sollte durch die beteiligten Gesundheitsdienstleister und Sektoren (v. a. Hausärztinnen/Hausärzte, Fachärztinnen/Fachärzte, Apothekerinnen/Apotheker, ambulant und stationär) gemeinsam festgelegt werden (wie dies in den Projekten teilweise erfolgt ist). Der Medikationsplan schafft prinzipiell mehr Transparenz zwischen den Gesundheitsdienstleistern, aber damit auch mehr Bedarf an Kommunikation. Die hierfür nötigen Kommunikationsmöglichkeiten sind zu schaffen. Damit diese akzeptiert werden, können Anforderungen jedoch nicht unabhängig von entsprechenden Rahmenbedingungen (Vergütung) festgelegt werden.

\section{Vorschlag 3: Technische Definition einer Gleichheit von Zeilen im eMP}

Aktuelle Situation. Aktuell sehen weder der BMP noch der eMP eine $\mathrm{Zu}$ sammenfassung mehrerer gleicher Zeilen $\mathrm{zu}$ einer einzigen Zeile im jeweiligen Medikationsplan vor. Deshalb werden im zeitlichen Verlauf insbesondere beim eMP jeweils neue Zeilen erstellt, es sei denn, die Gesundheitsdienstleister prüfen und bearbeiten diese entsprechend individuell. In PRIMA (und ARMIN) wurde als technische Implementierungshilfe eine sogenannte Zeilen-ID eingeführt. Zusammen mit einem definierten Regelwerk zur Handhabung der ID im Rahmen der Bearbeitung von Medikationsplanzeilen führt dies zur eindeutigen (Wieder-)Erkennung einer Me- dikationsplanzeile bei unterschiedlichen Gesundheitsdienstleistern. Darüber hinaus wird hiermit u.a. auch eine übersichtlichere Differenzdarstellung bei geänderten Inhalten ermöglicht.

Ziel. Erstellung und Evaluation eines definierten Regelwerks zur Handhabung gleicher Zeilen im BMP unter Zuhilfenahme einer Zeilen-ID (auch im Kontext der zu entwickelnden Standardprozesse aus Vorschlag 2).

\section{Vorschlag 4: Serverbasierter eMP}

Aktuelle Situation. Die Papierversion des BMP adressiert Patientinnen und Patienten. Diese wird nicht immer bei einer Arztvorstellung in der Praxis oder dem Krankenhaus mitgeführt. Oft wird eine nichtaktuelle oder unvollständige Version des BMP im Behandlungsprozess vorgelegt. Somit liegen zum Verordnungszeitpunkt trotz vorhandenem BMP keine validen Medikationsinformationen vor.

Ziel. Schließung dieser Informationslücke durch Schaffung einer serverbasierten Version des BMP entsprechend dem eMP (PRIMA) oder dem eMediPlan (MMP16), sodass die dort hinterlegte Version einem aktuellen und vollständigen BMP entspricht.

\section{Vorschlag 5: Lückenlose Integration der Kliniken durch serverbasierten eMP}

Aktuelle Situation. Zum Aufnahmezeitpunkt der Patientinnen und Patienten in den Kliniken wird häufig der papiergebundene BMP vorgelegt. Um diesen digital in das Krankenhausinformationssystem einzulesen, bedarf es eines 2-D-Barcodescanners. Dieser müsste an sehr vielen Orten in einem Klinikum verfügbar sein, da neben den Notaufnahmen auch in den Ambulanzen, Sprechstunden, $\mathrm{Pa}$ tientenaufnahmen elektiv, Stationen etc. Patientinnen und Patienten ,aufgenommen" werden. Zudem fehlen vor allem bei Notaufnahmepatienten aktuelle BMP.

Ziel. Um die Verfügbarkeit von aktuellen und vollständigen Medikationsinforma- 
tionen einer Patientin oder eines Patienten beim Sektorenübertritt von ambulant nach stationär oder auch umgekehrt sicherzustellen, wird es notwendig sein, neben der Papierversion für Patientinnen und Patienten eine professionelle Version des BMP zu definieren, welcher an die Ärztinnen und Ärzte sowie Apothekerinnen und Apotheker direkt adressiert und z. B. zusätzliche Informationen beinhaltet, wie Diagnosen und Indikation zur Medikation, und auch eine Historienrecherche zum Verlauf zulässt, um medikamentöse Gefährdungssituationen der Vergangenheit erkennen und vermeiden zu können. Diese Funktionen und deren Verfügbarkeit übersteigen die Möglichkeiten des Papiers und erfordern eine digitale Plattform, auf die die Gesundheitsdienstleister im Bedarfsfall zugreifen können.

\section{Fazit}

Der BMP ist ein wichtiger erster Schritt, um alle Gesundheitsdienstleister für AMTS zu sensibilisieren und einen notwendigen Kulturwandel im Umgang mit Arzneimitteln in der täglichen Routine einzuläuten. Die papiergebundene Version mit einem 2-D-Barcode wird vom Patienten sehr gut akzeptiert, ist aber für die Gesundheitsdienstleister nicht ausreichend. Dennoch ist der BMP die Basis, um Verantwortlichkeiten im Verordnungs- und Kommunikationsprozess zwischen allen Beteiligten klar zu regeln. Auch zukünftige digitale Medien oder servergebundene Lösungen werden nur dann erfolgreich sein, wenn diese Verantwortlichkeiten dann auch wahrgenommen werden.

\section{Korrespondenzadresse}

\section{Prof. Dr. med. H. Dormann}

Zentrale Notaufnahme, Klinikum Fürth Jakob-Henle-Str. 1, 90766 Fürth, Deutschland harald.dormann@klinikum-fuerth.de

Danksagung. Folgende Personen haben wesentlich zu den Projekten beigetragen: MetropolMediplan 2016 (MMP16): Frank Dörje, Bettina Plank-Kiegele, Manuela Linß, Christian Heidl, Martin Sedlmayr, Simone Schächtele, Susanne Schuster, Johanna Mathes, Zahra Abbas, Kristina Friedland, Hans-Ulrich Prokosch, Michael Bangemann, Werner Spiegl, Franz Jobst, Margit Schlenk, Claudia Röck, Jürgen Zerth,
Martin F. Fromm. Modellregion Erfurt: Natalie Bräuer, Dominic Fenske, Hagen Kellner, Harald Lapp, Marvin Münch, Jessica Papst. PRIMA: Miriam Felberg, Dirk Klintworth, Sabine Breiholz, Ann Kathrin Strunz, Hanna Seidling, Anette Lampert.

\section{Einhaltung ethischer Richtlinien}

Interessenkonflikt. H. Dormann, R. Maas, C. Eickhoff, U. Müller, M. Schulz, D. Brell, P.A. Thürmann geben an, dass kein Interessenkonflikt besteht.

Für alle drei Modellprojekte lagen positive Voten der zuständigen Ethikkommissionen und ein schriftliches Einverständnis der Projektteilnehmer vor. MetropolMediplan 2016: Das positive Votum wurde von der Ethikkommission der Friedrich-Alexander-Universität Erlangen-Nürnberg am 24.03.2015 erteilt. Modellregion Erfurt: Das positive Votum wurde von der Ethikkommission der Landesärztekammer Thüringen am 19.05.2015 erteilt. PRIMA: Das positive Votum wurde von der Sächsischen Landesärztekammer am 30.09.2015 erteilt. Diesem schloss sich die Ethikkommission der Landesärztekammer Thüringen am 11.12.2015 an

Open Access. Dieser Artikel wird unter der Creative Commons Namensnennung 4.0 International Lizenz (http://creativecommons.org/licenses/by/4.0/deed. de) veröffentlicht, welche die Nutzung, Vervielfältigung, Bearbeitung, Verbreitung und Wiedergabe in jeglichem Medium und Format erlaubt, sofern Sie den/die ursprünglichen Autor(en) und die Quelle ordnungsgemäß nennen, einen Link zur Creative Commons Lizenz beifügen und angeben, ob Änderungen vorgenommen wurden.

\section{Literatur}

1. Bundesministeriums für Gesundheit zur Verbesserung der Arzneimitteltherapiesicherheit (AMTS) (2010) Aktionsplan 2010-2012 des Bundesministeriums für Gesundheit zur Verbesserung der Arzneimitteltherapiesicherheit(AMTS) in Deutschland. https://www.akdae.de/AMTS/Aktionsplan/ Aktionsplan-2010-2012/index.html.Zugegriffen: 9. Febr. 2018

2. Bundesministeriums für Gesundheit zur Verbesserung der Arzneimitteltherapiesicherheit (AMTS) (2013) Aktionsplan 2013-2015 des Bundesministeriums für Gesundheit zur Verbesserung der Arzneimitteltherapiesicherheit (AMTS) in Deutschland. https://www.akdae. de/AMTS/Aktionsplan/Aktionsplan-2013-2015/ Aktionsplan-AMTS-2013-2015.pdf. Zugegriffen: 9. Febr. 2018

3. Aly F, Hellmann G, Moeller H (2013) Spezifikation für einen patientenbezogenen Medikationsplan, Version 2.0-für Modellvorhaben

4. Gesetz für sichere digitale Kommunikation und Anwendungen im Gesundheitswesen sowie zur Änderung weiterer Gesetze. Bundesgesetzblatt Jahrgang 2015, Teil 1 Nr. 54, Bonn 21. Dez. 2015. (http://www.bgbl.de/xaver/ bgbl/start.xav?startbk=Bundesanzeiger_BGBI\& jumpTo=bgbl115s2408.pdf). Zugegriffen: 9. Febr. 2018

5. Kassenärztliche Bundesvereinigung, GKV-Spitzenverband (2016) Artikel 1 Aufnahme eines $\S 29 a$ in den Bundesmantelvertrag-Ärzte, Bekanntmachungen, Deutsches Ärzteblatt 113(40). https://www.aerzteblatt.de/pdf.asp?id=182812 Zugegriffen: 7.Oktober 2016

6. Europäische Kommission (2009) Guideline on the readability of the labeling and package leaflet of medicinal products for human use. http://ec.europa.eu/health/files/eudralex/vol-2/ c/2009_01_12_readability_guideline_final_en. pdf (Erstellt: 12. Jan. 2009). Zugegriffen: 9. Febr. 2018

7. Botermann L, Krueger K, EickhoffC, Kloft C, Schulz M (2016) Patients' handling of a standardized medication plan: a pilot study and method development. Patient Prefer Adherence 10:621-630

8. Koordinierungsgruppe zur Umsetzung und Fortschreibung des Aktionsplanes zur Verbesserung der Arzneimitteltherapiesicherheit in Deutschland, Dr. Farid Aly, Dr. Gunther Hellmann, Dr. Horst Möller (2016) Spezifikation für einen bundeseinheitlichen Medikationsplan (BMP) gemäß § 31a SGB V. Anlage 3 zur Vereinbarung gemäß § 31a Abs. 4 Satz 1 SGB V über Inhalt, Struktur und Vorgaben zur Erstellung und Aktualisierung eines Medikationsplans sowie über ein Verfahren zur Fortschreibung dieser Vereinbarung. BMP Version 2.2,31. Mai 2016

9. Botermann L, Monzel K, Krueger K et al (2016) Evaluating patients' comprehensibility of a standardized medication plan. Eur J Clin Pharmacol 72:1229-1237

10. Botermann L, Wachter A, Monzel K et al (2017) Do patients with diabetes type 2 or chronic heart failure understand a medication plan? Pharmazie 72:764-768

11. Dormann $H$, Maas R, Langer M, Mathes J, Jobst F (2014) eMediPlan - ein digitales Infrastrukturprojekt zum einheitlichen Medikationsplan für Deutschland. In: Duesberg F, Hellmann G (Hrsg) Health 2014 - Sonderausgabe Medikationsplan / AMTS - Special Edition Medication List / Medication Safety - Édition Spécial Plan de Médication / Sécurité des Traitments Médicamenteux. Medical future Verlag, Solingen, S104-107

12. Bundesministeriums für Gesundheit zur Verbesserung der Arzneimitteltherapiesicherheit (AMTS) (2016) Aktionsplan 2016-2019 des Bundesministeriums für Gesundheit zur Verbesserung der Arzneimitteltherapiesicherheit (AMTS) in Deutschland. https://www.akdae. de/AMTS/Aktionsplan/Aktionsplan-2016-2019/ Aktionsplan-AMTS-2016-2019.pdf. Zugegriffen: 16. Febr. 2018 